${ }^{1}$ Centro de Educación Médica, Escuela de Medicina Pontificia Universidad Católica de Chile. Santiago, Chile.

2Dirección de Postgrado, Escuela de Medicina Pontificia Universidad Católica de Chile. Santiago, Chile. ${ }^{3}$ Departamento de Psiquiatría, Escuela de Medicina Pontificia Universidad Católica de Chile, Santiago, Chile.

aBioquímica, PhD en Farmacología. bPsicóloga, Magíster en Psicología Educacional.

Fuente de apoyo financiero: Fondo Nacional de Desarrollo Científico y Tecnológico FONDECYT (proyecto \# 1150340; otorgado a MB).

Autores no declaran conflictos de interés.

Recibido el 3 de julio de 2017, aceptado el 18 de noviembre de 2017.

Correspondencia a: Marcela Bitran PhD. Centro de Educación Médica, Escuela de Medicina Pontificia Universidad Católica de Chile. Diagonal Paraguay 362, segundo piso. Santiago, Chile. mbitran@med.puc.cl

\title{
Preocupación por el bienestar de residentes, un tema presente en la Conferencia Latinoamericana en Educación de Residentes (LACRE) 2017
}

\author{
MARCELA BITRAN ${ }^{1, a}$, MATÍAS GONZÁLEZ ${ }^{2}$, PÍA NITSCHE³, \\ DENISSE ZÚÑIGA ${ }^{1, \mathrm{~b}}$, ARNOLDO RIQUELME ${ }^{1}$
}

\section{Concern for residents' wellbeing, an issue discussed at the latin american conference on resident education (LACRE) 2017}

At the end of May 2017, the third version of the Latin American Conference on Resident Education, LACRE, was held in Chile; it convened 433 people from 14 regional countries. Chronic stress and emotional exhaustion of residents was one of the topics discussed. Reports from different countries documented that about half of residents suffer from burnout. This is, they are emotionally drained, indifferent towards their patients and with a sense of low personal fulfillment at work. This article describes the contributions presented in LACRE about interventions or institutional programs designed to reduce burnout and promote self-care of residents. The relevance of these initiatives is discussed in the current global context, considering the available evidence on the effectiveness of interventions to promote well-being among residents. International experts are making renewed and eloquent calls to medical educators and organizations to get involved in the solution of the erosion of resident wellbeing during the residence.

(Rev Med Chile 2017; 145: 1330-1335)

Key words: Burnout, Professional; Chile; Education, Medical; Physicians; Self Care.

A fines de mayo de 2017 se realizó la tercera versión del LACRE (Latin American Conference on Resident Education) en el que participaron 433 personas entre residentes y académicos de 14 países de la región. Tres simposios, 21 talleres, 30 presentaciones orales y 107 posters resumieron la experiencia de líderes académicos, educadores médicos, y estudiantes que asistieron a la conferencia. Este encuentro, el tercero desde 2013, es fruto de la colaboración entre el Royal College of Physicians and Surgeons of Canada, la Pontificia Universidad Católica de Chile y la Organización Panamericana de Salud (OPS). Nació con el propósito de constituir un foro para las américas, orientado a quienes estén involucrados en el proceso de formación de residentes de especialidades médicas y de otros profesionales de las carreras de la salud ${ }^{2}$.

Uno de los temas discutidos en esta tercera versión del LACRE, y que plantea un continuo desafío para la comunidad de educadores médicos es el estrés crónico y desgaste emocional que sufren los residentes debido a las altas demandas asociadas a su formación.

El Summit de ICE (International Clinical Educators) inauguró la reflexión con el provocativo título "Let's educate with the mind and the heart: How do we teach without breaking the student?" En los dos días siguientes los asistentes a la conferencia tuvieron oportunidad de participar de diversos 
talleres relacionados con el tema: Promoción de Bienestar, Mejoría de Ambiente Educacional, Enfrentamiento del Residente en Dificultades, Inteligencia Emocional y Desarrollo de Programas de Bienestar para Residentes.

Las presentaciones orales y posters trajeron testimonios de educadores de Argentina ${ }^{3}$, Chile $e^{4,5}$, Colombia $^{6}$, Ecuador ${ }^{7}$, El Salvador ${ }^{8}$, México $^{9-11}$, Nicaragua $^{12}$ y Paraguay ${ }^{13,14}$ que confirman que cerca de $50 \%$ de los residentes encuestados padece del síndrome de desgaste laboral o burnout. Una representación visual de los conceptos más frecuentes abordados en dichos trabajos se presenta en forma de nube de palabras (Figura 1).

\section{Burnout durante la residencia: algunas reflexiones}

Las personas que sufren burnout se sienten drenadas emocionalmente, se comportan con desafección e indiferencia hacia los demás y tienen un bajo aprecio por el propio desempeño laboral ${ }^{15}$. En personas altamente motivadas por su trabajo, como los residentes y médicos en general, el burnout puede coexistir inicialmente con la empatía, la satisfacción personal y el cuidado y preocupación por los demás. Sin embargo, el hecho de estar expuesto a un estrés crónico e inescapable, asociado a un trabajo extenuante suele terminar con estos atributos ${ }^{16}$. En palabras de Cristina Maslach, pionera en el estudio de este síndrome, "Burnout is an erosion in values, dignity, spirit, and will” (citado en Epstein, 2016 $6^{16}$ ).

Diversos estudios demuestran que el burnout conlleva un alto riesgo no solo para la salud de los residentes sino también para el cuidado de sus pacientes, debido al aumento de los errores médicos asociados a este síndrome ${ }^{17,18}$. La erosión del bienestar de los médicos durante la residencia es un problema difícil de resolver pues el residente tiene una condición dual de aprendiz y médico tratante, que lo expone a situaciones de alta responsabilidad con insuficiente experiencia y limitada autonomía ${ }^{19,20}$. Cuando este desbalance es muy grande o dura demasiado, termina por socavar la motivación intrínseca del estudiante, quien queda atrapado en una situación desempoderada: se encuentra sin suficiente poder de decisión (insuficiente autonomía), debe hacerse cargo de un resultado sub óptimo o negativo (baja autoeficacia), y es objeto del juicio de sus profesores y pares (se resiente su relación con los otros).

A este escenario inestable se suman factores sociológicos que hacen que hoy en día la conexión y comunicación del residente con los docentes clínicos y demás miembros del equipo médico sea más desafiante. Las diferencias generacionales entre docentes y residentes se expresan -entre otros-en distintas formas de relacionarse con el trabajo y la $v_{i d a}{ }^{21}$. Las nuevas generaciones de profesionales otorgan un valor creciente al bienestar personal y esperan que sus trabajos sean compatibles con la vida y salud personales ${ }^{21}$. Estas diferencias generacionales están muchas veces a la base de los juicios recíprocos negativos e incomunicación. No es infrecuente escuchar a los docentes quejarse de la 'flojera' o 'irresponsabilidad' del residente actual, o a los residentes, hablar de la 'rigidez' y 'autoritarismo' del docente.

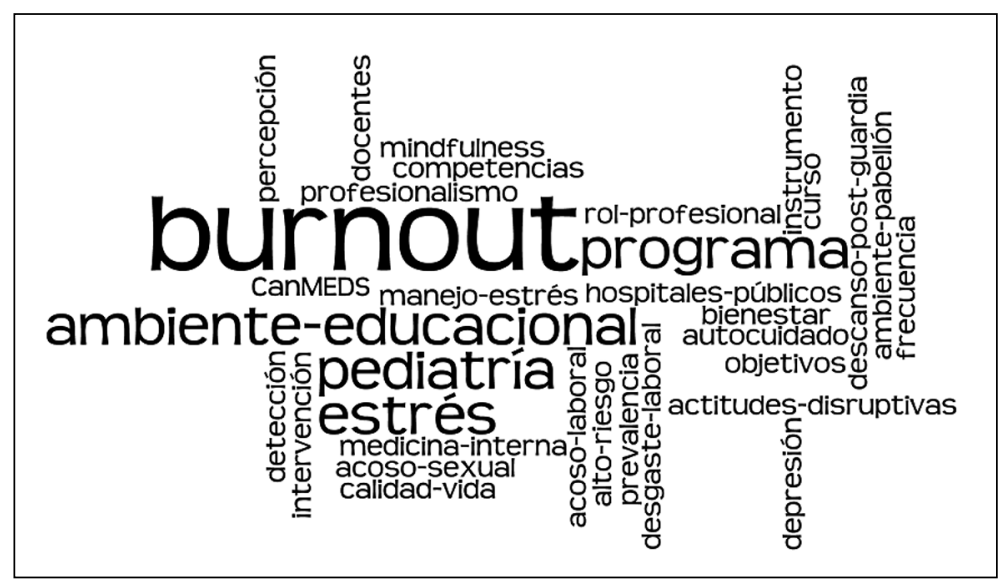

Figura 1. Nube de palabras obtenida de los títulos de los 18 trabajos presentados al LACRE 2017 referentes a bienestar de los residentes. El tamaño de la letra es proporcional a la frecuencia de cada palabra. 
Por otra parte, la cultura médica hace difícil que los estudiantes (y los médicos en general) reconozcan sus errores y problemas personales. Así, las probabilidades que un 'residente en problemas' pida ayuda a tiempo se ven reducidas por temor al estigma asociado al burnout y otras enfermedades psicológicas $^{22}$.

Por todas estas razones, combatir el burnout en la residencia es un desafío más complejo que en otros ambientes laborales o educacionales.

\section{¿Es posible revertir esta situación? ¿Qué dice la evidencia?}

Por cierto, el burnout no es exclusivo de los programas de residencia en Latinoamérica, ni tampoco de esta etapa de la formación médica. De hecho, más de 20 años de investigación documentan la gravedad de la situación tanto en médicos residentes como en profesionales médicos en Norteamérica y Europa ${ }^{23,24}$. Y la situación no parece mejorar: uno de los estudios más robustos, que involucró a más de 7.000 médicos estadounidenses reveló que, entre 2011 y 2014 , la frecuencia de burnout subió de $45,5 \%$ a $54,4 \%{ }^{25}$.

La primera revisión sistemática y metaanálisis de la efectividad de las intervenciones para reducir el burnout y promover el bienestar en residentes y médicos fue publicada en noviembre de 2016, en la revista Lancet ${ }^{26}$. Tait Shanafelt y su equipo de la Clínica Mayo, líderes en la investigación en burnout, revisaron 2.617 artículos y seleccionaron 15 estudios randomizados y 37 estudios de cohorte, la gran mayoría de países de altos ingresos. Los resultados de este y otros metaanálisis más recientes ${ }^{27}$ coinciden en que tanto las intervenciones enfocadas en los individuos como las intervenciones organizacionales pueden reducir el riesgo de burnout en médicos y residentes, particularmente el riesgo de agotamiento emocional. Dentro de las intervenciones individuales o de grupo pequeño se cuentan talleres de manejo del estrés y autocuidado, talleres de comunicación efectiva, y cultivo del bienestar mediante intervenciones basadas en prácticas de mindfulness ${ }^{26}$. Las intervenciones organizacionales comprenden disminución de la carga horaria, reestructuraciones de los turnos y entrenamiento de habilidades de comunicación y trabajo colaborativo de los equipos de salud ${ }^{26,27}$.

\section{Un llamado a la acción}

Recientemente un colectivo de connotados educadores médicos, líderes académicos e investigadores expertos en burnout hizo un llamado urgente a la comunidad de educadores médicos y autoridades de salud a actuar para mejorar el bienestar de los especialistas en formación y mitigar su burnout ${ }^{28}$.

Este grupo, llamado Collaborative for Healing and Renewal in Medicine (CHARM), está integrado por académicos de Icahn School of Medicine at Mount Sinai, University of Rochester Medical Center, Mayo Clinic, Weill Cornell Medical College, Harvard Medical School y Massachusetts General Hospital. Su llamado a la acción, publicado en el número de mayo de 2017 de la prestigiada revista Academic Medicine ${ }^{28}$, plantea un marco de recomendaciones multinivel que incluye a individuos, programas, hospitales y países tendientes a promover el bienestar y disminuir el burnout de los residentes. La idea es que los cambios realizados a estos distintos niveles ataquen la raíz del problema y promuevan una cultura donde los estudiantes puedan aprender en espacios que los nutran a lo largo de sus carreras y no que los lesionen. Para ello, postulan los autores, es necesario desarrollar un 'menu' de opciones de bienestar que responda a las necesidades de la variedad de aprendices y que permita estandarizar el esfuerzo de disminuir el burnout ${ }^{28}$.

\section{¿Qué pasa en Latinoamérica? Lo que se dijo en el LACRE}

Aunque la mayoría de los estudios presentados en el LACRE 2017 relativos al bienestar se remitió a reportar la frecuencia de burnout y depresión, algunos mostraron intervenciones focalizadas $o$ programas institucionales diseñados para disminuir el burnout y fomentar el autocuidado de los residentes.

Por ejemplo, la Escuela de Medicina del Instituto Tecnológico y de Estudios Superiores de Monterrey, México, presentó un programa para crear un ambiente educacional libre de maltrato que incluye talleres de estudio y discusión entre pares $^{8}$, y la implementación de un sistema en línea para que los residentes reporten incidentes críticos relativos al ambiente educacional sin temor de represalias ${ }^{29}$. 
Por otra parte, el Programa General de Bienestar de Residentes de la Pontificia Universidad Católica de Chile, a cargo de profesionales del área de la salud mental combina múltiples iniciativas a nivel individual y organizacional: Evaluación de las condiciones de vida de los residentes y clima educacional ${ }^{4}$, trabajo personal y consejería integral, trabajo grupal para fortalecer competencias y promover factores protectores al estrés, consejería espiritual, desarrollo de instancias de formación para los docentes en prevención de problemas de Salud Mental y creación de un conjunto de cursos para residentes para fomentar el autocuidado, incluyendo el cultivo de mindfulness ${ }^{30}$.

Por su parte, la Escuela Posgrados de la Facultad Ciencias de la Salud de la Universidad Dr. Matías Delgado de El Salvador implementó un programa de apoyo a la salud de los residentes mediante sesiones de terapia de apoyo grupal e individual con médicos psiquiatras y psicólogos para el manejo del estrés y conflictos, con el objeto de reducir la deserción de sus residentes ${ }^{8}$.

Estos son algunos ejemplos de iniciativas regionales que demuestra un compromiso institucional para la solución de este problema. A la base de estas iniciativas está el reconocimiento que el burnout no es un problema exclusivo del individuo sino que atañe también a las organizaciones formadoras.

\section{Conclusiones}

Pese a su corta historia ${ }^{2}$, el LACRE (Latinoamerican Conference on Resident Education) se ha constituido en un dinámico foro Latinoamericano de encuentro para líderes académicos, educadores médicos y estudiantes de especialidades médicas y de otras profesiones de la salud. Así lo indica el éxito de su tercera versión realizada el pasado mes de mayo en Santiago de Chile. La asistencia, número y diversidad de talleres ofrecidos, así como en el número de posters y presentaciones orales presentadas reflejan la madurez y el grado de complejidad alcanzado por el LACRE en su tercera versión.

En dicho encuentro quedó en evidencia la preocupación de los educadores médicos de distintos países de la región por el bienestar de residentes. Las cifras de burnout en Latinoamérica son tan preocupantes como en el resto del mundo: prác- ticamente la mitad de residentes se encuentra en un estado de agotamiento emocional, despersonalización y baja satisfacción con su desempeño profesional $^{4-14}$, situación que implica riesgos para los afectados como también para sus pacientes ${ }^{17,18}$.

La tradicional respuesta asistencial de las organizaciones educacionales al 'residente en problemas' es insuficiente y deja en claro que hasta ahora hemos concebido este tema como un problema de salud mental individual. Sin embargo, las altísimas cifras de residentes con burnout y un somero análisis de la 'arquitectura' de esta etapa de formación dejan en claro que la erosión del bienestar de los residentes es el resultado de un problema sistémico que nos compete como educadores y líderes médicos. El burnout no es un problema exclusivo del residente; representa una fisura entre el residente y su ambiente laboral ${ }^{15}$.

¿Es posible repensar la residencia de modo que la formación de especialistas no ocurra a costa del bienestar y salud personales? ¿Podremos formar profesionales capaces de velar por su autocuidado así como por el cuidado de sus pacientes? O por el contrario, ¿debemos aceptar que el sufrimiento y desgaste son inseparables de la especialización médica? ¿Parece sensato esperar que la generación actual de estudiantes soporte las mismas dificultades que sus maestros, que estudiaron en condiciones aún más difíciles y recibieron un trato más estricto?

El análisis científico de más de dos décadas de investigación indica que es posible revertir parcialmente esta situación de desgaste de residentes y profesionales de la salud mediante intervenciones individuales que dan herramientas de autocuidado, como también gracias a intervenciones organizacionales que promueven un mejor clima y condiciones laborales ${ }^{26,27}$.

Connotados expertos en el tema insisten que ya es tiempo que cambiemos el discurso del burnout al bienestar ${ }^{28,31-32}$; para lo cual es preciso reconocer que nos cabe una responsabilidad en el desgaste de nuestros estudiantes y que tenemos una obligación ética para con ellos y sus pacientes ${ }^{15}$.

En la Conferencia Latinoamericana en Educación de Residentes LACRE, realizada en mayo de 2017 en Santiago de Chile, supimos de programas de distinta complejidad orientados a promover el bienestar y autocuidado de los residentes. Estas son iniciativas incipientes y están a una distancia considerable de los programas de bienestar de cen- 
tros de educación médica líderes internacionales como Clínica Mayo $^{33}$ o la Escuela de Medicina de la Universidad de Stanford ${ }^{31}$. Sin embargo, su desarrollo revela que existe una conciencia creciente en la comunidad de educadores médicos de la región acerca de la responsabilidad que nos cabe en este tema. Porque nos compete, porque somos parte del problema, debemos ser parte de la solución y diseñar programas que apunten a reducir el desgaste y agotamiento emocional de nuestros residentes.

\section{Referencias}

1. Sitio oficial de la conferencia LACRE 2017. http://www. lacre.cl/spa/

2. Heusser F, Riquelme A. Primer Congreso Latinoamericano de Formación de Residentes en Especialidades Médicas. Rev Chil Pediatr 2014; 85 (2): 139-43.

3. Ladenheim R, Ledesma L, Valentini R, Cusumano A. Implementación del descanso post guardia en la residencia de medicina interna: percepción de residentes y docentes. Investigación en Educación Médica 2017; 6 (22): e9-e10. https://doi.org/10.1016/j.riem.2017.01.026.

4. González M, Silva MP, Grez M, Hoyl T. Residentes con alto riesgo de burnout: Detección e intervención. Investigación en Educación Médica 2017; 6 (22): 137-8. https://doi.org/10.1016/j.riem.2017.01.141.

5. Irribarra L, Campos M, Klaber I, Irarrazaval S, Lira MJ, Mery P, et al. Calidad de vida y burnout en residentes de las distintas especialidades. Investigación en Educación Médica 2017; 6 (22): e43. https://doi.org/10.1016/j. riem.2017.01.104.

6. Mulett-Vásquez E, Castano-Ramírez OM, Pérez-Arias LF. Prevalencia de depresión en estudiantes de posgrados médicos y los factores asociados. Investigación en Educación Médica 2017; 6 (22): e48. https://doi. org/10.1016/j.riem.2017.01.115

7. Torres-Herrera C, Puga-Tejada M, Rodríguez-Morales K, Bermeo-Soto B, Arriciaga-Nieto H, Oleas R. Effect of educational environment of ecuadorian residency training programs in professional burnout syndrome presence in postgraduate residents. Investigación en Educación Médica 2017; 6 (22): 133. https://doi.org/10.1016/j.riem.2017.01.131.

8. Flores Muñoz RJ, Quiteño C, Lara R, Figueroa M, Bello M, Calix JJ, et al. Programa manejo del estrés en médicos residentes. Investigación en Educación Médica 2017; 6 (22): e7. https://doi.org/10.1016/j.riem.2017.01.020

9. Cordero Díaz MA, Guerra D, Arce CF, Dávila Rivas JA.
Programa de profesionalismo y bienestar para médicos residentes. Investigación en Educación Médica 2017; 6 (22): e37. https://doi.org/10.1016/j.riem.2017.01.090.

10. Martínez JA, de la Cruz Cruz RA, Rocha Flores DE. Uso de un instrumento validado para medir el estrés - desgaste laboral en médicos residentes de la especialidad de Pediatría en hospitales públicos y privados al noreste de México. Investigación en Educación Médica 2017; 6 (22): 138-9. https://doi.org/10.1016/j. riem.2017.01.143.

11. Palmer-Morales Y, Prince-Vélez R, Medina-Ramírez MCR, López-Palmer DA. Frecuencia de rasgos de depresión en médicos internos de pregrado del Instituto Mexicano del Seguro Social en Mexicali, Baja California. Investigación en Educación Médica 2017; 6 (22): 75-9. https://doi.org/10.1016/j.riem.2016.08.001.

12. Romero T, Hernández R. Estrés laboral en médicos residentes de los hospitales públicos de Managua, Nicaragua, abril a mayo 2016. Investigación en Educación Médica 2017; 6(22):e2. https://doi.org/10.1016/j. riem.2017.01.009.

13. Martínez Álvarez GdJ, Godoy Sánchez L, Brizuela Rodas MB, Rodas de Brizuela NC. Prevalencia de síndrome de burnout en residentes de pediatría. Un estudio multicéntrico. Investigación en Educación Médica 2017; 6 (22): e45. https://doi.org/10.1016/j.riem.2017.01.108.

14. Martínez Álvarez GdJ, Godoy Sánchez L, Fernández Dipp CC, Chamorro Caballero ZE. Prevalencia y variación del síndrome de burnout en residentes de pediatría. Investigación en Educación Médica 2017; 60; 6 (22): e27. https://doi.org/10.1016/j.riem.2017.01.067.

15. Maslach C, Leiter M. New insights into burnout and health care: Strategies for improving civility and alleviating burnout. Medical Teacher 2017; 39 (2): 160-3.

16. Epstein RM, Privitera MR. Doing something about physician burnout. Lancet 2016; 388 (10057): 2216-7. doi: 10.1016/S0140-6736(16)31332-0.

17. Shanafelt TD, Balch CM, Bechamps G, Russell T, Dyrbye L, Satele D, et al. Burnout and Medical Errors Among American Surgeons. Ann Surg [Internet]. 2010 Jun [citado el 11 de marzo de 2017]; 251 (6): 995-1000. Available from: http://www.ncbi.nlm.nih.gov/pubmed/19934755.

18. Fahrenkopf A, Sectish T, Barger L, Chiang V, Edwards S, Wiedermann B, et al. Rates of medication errors among depressed and burnt out residents: prospective cohort study. BMJ [Internet]. 2008 [citado el 8 de marzo de 2017]; 336. Available from: http://www.bmj.com/content/bmj/336/7642/488.full.pdf.

19. Ratanawongsa A, Wright SM \& Carrese JA. Well-being in residency: a time for temporary imbalance? Medi- 
cal Education 2007; 41: 237-80. doi:10.1111/j.13652929.2007.02687.x

20. Hoffman B. Using self-determination theory to improve residency training: learning to make omelets without breaking eggs. Acad Med 2015; 90 (4): 408-10. doi: 10.1097/ACM.0000000000000523.

21. Eckleberry-Hunt J, Tucciarone J. The challenges and opportunities of teaching "generation y" J Grad Med Educ 2011; 3(4): 458-61. doi: 10.4300/JGME-03-04-15.

22. Dyrbye L, Eacker A, Durning SJ, Brazeau C, Moutier C, Massie S, et al. The impact of stigma and personal experiences on the help-seeking behaviors of medical students with burnout. Acad Med 2015; 90 (7): 961-9. doi: 10.1097/ACM.0000000000000655.

23. Dyrbye LN, West CP, Satele D, Boone S, Tan L, Sloan J, et al. Burnout among U.S. medical students, residents, and early career physicians relative to the general U.S. population. Acad Med [Internet] 2014; 89 (3): 443-51. Available from: http://www.ncbi.nlm.nih.gov/pubmed/24448053.

24. Lee RT, Seo B, Hladkyl S, Lovell BL, Schwartzmann L. Correlates of physician burnout across regions and specialties: A meta-analysis. Hum Resour Health 2013 doi: 10.1186/1478-4491-11-48.[PMC free article] [PubMed] [Cross Ref].

25. Shanafelt TD, Hasan O, Dyrbye LN, Sinsky C, Satele $\mathrm{D}$, Sloan J, et al. Changes in Burnout and Satisfaction With Work-Life Balance in Physicians and the General US Working Population Between 2011 and 2014. Mayo Found Med Educ Res n Mayo Clin Proc 2015; 90 (12): 1600-13.

26. West CP, Dyrbye LN, Erwin PJ, Shanafelt TD. Interventions to prevent and reduce physician burnout: a systematic review and meta-analysis. Lancet 2016; 388 (10057): 2272-81. doi: 10.1016/S0140-6736(16)31279-X. Epub 2016 Sep 28.

27. Panagioti M, Panagopoulou E, Bower P, Lewith G, Kontopantelis E, Chew-Graham C, et al. Controlled Interventions to Reduce Burnout in Physicians: A Systematic Review and Meta-analysis. JAMA Intern Med 2017; 177 (2): 195-205. doi: 10.1001/jamainternmed.2016.7674.

28. Ripp JA, Privitera MR, West C, Leiter R, Logio L, Shapiro J, et al. Well-Being in Graduate Medical Education: A Call for Action. Acad Med 2017 May 2. doi: 10.1097/ ACM.0000000000001735.

29. Cordero AM, Arce C, Dávila JA. Promoting an Optimal Clinical Learning Environment for Residency Education and Patient Care. Investigación en Educación Médica 2017, 6 (22): 135.

30. Nitsche MP, Bitran M, González M. ¿Pueden los objetivos de un curso de mindfulness tributar a competencias del rol profesional CanMEDS relacionadas con el autocuidado? Investigación en Educación Médica 2017; 6 (22): 131-2. https://doi.org/10.1016/j.riem.2017.01.128.

31. Salles A, Liebert CA, Greco RS. Promoting Balance in the Lives of Resident Physicians: A Call to Action. JAMA Surg 2015; 150 (7): 607-8. doi: 10.1001/jamasurg.2015.

32. Haramati A, Cotton S, Padmore JS, Wald HS, Weissinger PA. Strategies to promote resilience, empathy and well-being in the health professions: Insights from the 2015 CENTILE Conference. Med Teach 2017; 39 (2): 118-9. doi: 10.1080/0142159X.2017.1279278.

33. Mayo Clinic Physicians Wellbeing Program. http://www. mayo.edu/research/centers-programs/physician-well-being-program/overview. 